\title{
La enseñanza de español y la formación del docente de español como lengua extranjera ${ }^{1}$
}

\author{
Pedro Barros García \\ Facultad de Filosofía y Letras \\ Universidad de Granada
}

Recibido: 16 febrero 2005 / Versión aceptada: 15 marzo 2005

ISSN: $1697-7467$

\begin{abstract}
RESUMEN: Este trabajo tiene como objetivo orientar a quienes quieren dedicarse a la tarea docente. Haremos, al principio, unas breves reflexiones sobre cómo aprendemos una lengua y sobre los factores que condicionan la elección del enfoque que queremos seguir. Damos después una breve referencia a la enseñanza del E/LE y a su situación curricular en las Facultades de Letras.

La parte fundamental del trabajo va destinada a la formación de los docentes de E/LE, haciendo especial hincapié en un aspecto que ha despertado un inusitado interés en los trabajos más recientes “el pensamiento del profesor”, que tendrá una importante repercusión en el manejo de la clase.

Palabras clave: enseñanza de lenguas extranjeras; enseñanza de E/LE; papel del profesor; pensamiento del profesor.
\end{abstract}

\begin{abstract}
The aim of this paper is to give directions to anyone who wants to develop his/her career as a language teacher. On the one hand, we introduce some brief reflections on how a language is learnt and on the factors which determine the approach we want to follow. In addition, we give a brief reference of S/SL teaching and its situation in the faculties' study plans.

This essential part of this study explores some aspects of the S/SL teacher's thinking, an important component of S/SL teachers' education. As we will see, this issue may have important consequences on class-management.

Key words: foreign language teaching; S/SL teaching; teacher's role; teacher's thoughts
\end{abstract}

\section{INTRODUCCIÓN}

En este trabajo, queremos aportar algunas reflexiones sobre la labor del profesor de español como lengua extranjera; labor compleja que ha ido evolucionando al compás de las diferentes metodologías empleadas en la enseñanza de las lenguas; el profesor de hoy no sólo tiene que ser un buen conocedor de los recursos lingüísticos de la lengua que enseña, sino

1 Este trabajo fue presentado como ponencia en las "Jornadas de Lengua Española. Las profesiones del filólogo", celebradas en la Universidad de Valencia, del 16 al 18 de noviembre de 2004, y organizadas por el grupo Val. Es. Co. del Departamento de Filología Española. 
que también ha de mostrar grandes dotes como sociólogo, psicólogo, etnógrafo, actor e incluso diplomático. Con esto no quiero asustar a quienes tengan la intención de dedicarse a esta compleja profesión, sino más bien animarles a prepararse concienzudamente, para que después encuentren la satisfacción que produce el trabajo bien hecho y el reconocimiento y gratitud de los buenos discípulos.

Alguien ha comparado la enseñanza con un juego de malabarismo: Si quieres mantener en el aire unas cuantas bolas, cuesta un tiempo conseguir que no se caigan, e incluso cuando ya tienes mucha práctica, puedes complicar el juego añadiendo alguna bola más. Pues algo parecido sucede en la enseñanza: los profesores noveles se centran, al principio, en un par de cosas (¿qué actividades puedo programar?, ¿cómo puedo motivar a los alumnos?, ¿qué tiempo necesitan para realizar las tareas?, etc.), en tanto que los profesores experimentados, al igual que el malabarista con mucha práctica, es capaz de combinar simultáneamente diferentes técnicas y actividades. Saber enseñar, saber conducir una clase, requiere que entren en juego al mismo tiempo un gran número de bolas. Vamos ahora a analizar el papel de algunas de ellas.

\section{2. ¿Cómo aprendemos una lengua?}

Seguramente, quienes tengan ya experiencia en el aprendizaje de alguna lengua podrán recordar algunos aspectos concretos, como, por ejemplo, responder a alguna de estas preguntas: ¿Cuál era el método utilizado?, ¿qué principios teóricos regían el aprendizaje?, ¿cómo se presentaban los contenidos en el libro de texto?, ¿qué destrezas se practicaban?, ¿qué tipos de actividades o de ejercicios se hacían?, ¿cuál era el papel del profesor y de los estudiantes?, ¿qué tipo de materiales se empleaban?, ¿cómo se hacía la evaluación del aprendizaje?, etc. No agotamos aquí la lista de preguntas posibles, pero creo que son suficientes para que nos planteemos algunas cuestiones: ¿Estamos de acuerdo con aquel enfoque, con el diseño de las tareas para lograr los objetivos previstos y con los procedimientos empleados? ¿Estaríamos dispuestos a repetir esos mismos planteamientos e imitar el papel de aquel profesor en nuestras tareas docentes? Pretendemos reflexionar sobre estos aspectos, para tratar de concretar la labor del docente de lengua extranjera.

\section{FaCtores que determinan la UTILIZACión de NUEvos ENFOQUES}

No pretendemos hacer aquí una historia de la evolución de los distintos métodos utilizados en la enseñanza de lenguas - existe ya una amplia bibliografía que puede consultarse -, sino tratar de explicar el porqué de la aparición constante de nuevos enfoques. Todos nacen con la pretensión de superar las deficiencias encontradas en los precedentes y aportar nuevas teorías que puedan explicar o dar respuesta a la pregunta ¿cómo aprendemos? G. Neuner y H. Hanfeld, (1993: 9) presentan un esquema que agrupa los factores que influyen en el desarrollo de un nuevo método, cuya adaptación, hecha por Pilar Melero (2000: 10), ofrecemos a continuación: 
Las propuestas procedentes

de la pedagogía y de la

investigación didástica.
La aplicación de las aportaciones procedentes de la psicología del aprendizaje y de la psicologia genética.
La forma de enseñar en la clase de lengua materna (por ejemplo, lengua española en el sistema escolar español).
Los objetivos concretos del curso (por ejemplo: español para turistas, para inmigrantes).
Los modos de enseñanza y aprendizaje del propio país.

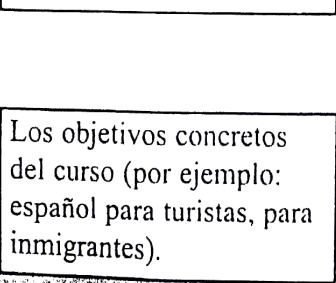

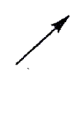

En los métodos/enfoques de enseñanza de lenguas extranjeras influye/-n:

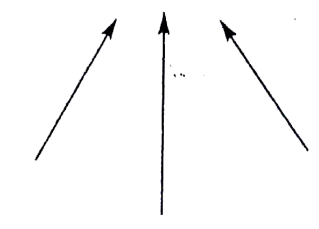

Ideas procedentes del trabajo con textos literarios.
Las repercusiones de las nuevas aportaciories de la lingüistica.

Los conceptos e ideas que se tienen del país o paises donde se hábla la lengua extranjera.
Las condiciones individuales de aprendizaje y las características específicas del grupo.

Las experiencias del proceso de aprendizaje de otra lengua extranjera diferente.

Todos estos factores -y algunos más que están implícitos, como los socioculturales, contextuales, en definitiva, pragmáticos- están relacionados entre sí y se influyen mutuamente, dando lugar a nuevos enfoques metodológicos que evolucionan en la medida que se van produciendo avances en las teorías de aprendizaje, en los planes de estudio, en la autonomía del aprendizaje y en los cambios políticos y sociales.

En las tres últimas décadas, la enseñanza de las lenguas extranjeras se ha interesado por conocer campos ajenos a los de la lingüística aplicada, en un afán por encontrar respuesta a muchos de los problemas no resueltos por los sucesivos modelos de descripción linguiística 
utilizados. Desde una primera orientación estructuralista, basada en las teorías conductistas (Skinner, años 60) de estímulo respuesta, siguiendo con los modelos cognitivos y humanistas, de inspiración generativista, hasta los más recientes enfoques comunicativos, centrados en el desarrollo del individuo y que han puesto el énfasis en los factores afectivos y en el desarrollo de la dimensión sociocultural y pragmática.

La utilización y desarrollo de cualquiera de estos modelos (gramática-traducción, directo, situacional, audiovisual, nocio-funcional, enfoque por tareas, procesuales, interculturales, etc.) estará determinado por las teorías lingüísticas sobre el aprendizaje de lenguas en vigor y por la formación y los condicionamientos circunstanciales de los profesores. Para tener una visión de conjunto sobre los datos históricos relacionados con la enseñanza del español como lengua extranjera se puede consultar, entre otros, el amplio trabajo del profesor Aquilino Sánchez (1992) o el práctico y documentado trabajo de Pilar Melero (2000), donde se hace un repaso de las distintas tradiciones, corrientes y métodos que, desde el siglo XVI hasta nuestros días, han orientado este tipo de enseñanza.

\section{La enseñanza del E/LE en EsPaña}

En España, como sucedía también en otros países, la enseñanza de la lengua se centraba en la descripción del sistema lingüístico, en el conocimiento de su estructura, sin tener en cuenta su función como instrumento de comunicación, necesaria para saber utilizar nuestra lengua en las distintas situaciones contextuales y en las relaciones internacionales.

Las circunstancias históricas serán las que determinarán el cambio de rumbo en la enseñanza del Español: el fin del aislamiento de nuestro país a partir del ingreso en la ONU (1955), el auge turístico a partir de los sesenta, el ingreso en la Comunidad Económica Europea (1986), generan un interés inusitado por la lengua y la cultura españolas. Si añadimos a esto el aumento de la población que utiliza el español como lengua materna (unos 400 millones), que la convierten en la segunda lengua internacional, por número de hablantes, hace que cada vez sea mayor el número de personas, de todo el mundo, que se interesen por aprender nuestra lengua. Como consecuencia de ese interés se incrementa cada año el número de centros dedicados a la enseñanza de nuestro idioma, tanto dentro como fuera de nuestras fronteras: Escuelas de idiomas, Academias, Centros de Lenguas dependientes de las universidades, como el CLM de la universidad de Granada, nuevos Departamentos de Español en las Universidades extranjeras. Paralelamente, también se amplía la demanda de profesores, bien preparados, que lo enseñen o que presten sus servicios en editoriales, centros o empresas dedicadas a la traducción de documentos, asesorías lingüísticas en embajadas y consulados, etc.

En este sentido, es de destacar el enorme papel que está desarrollando el Instituto Cervantes, desde su creación en 1991, tanto en la difusión de la lengua y cultura españolas, abriendo sedes en las principales ciudades del mundo - según mis datos, unas 36 en la actualidad - como en la labor de formación de profesores, fomentando y apoyando los cursos de Metodología por todo el país, en la actualización de los métodos, estrategias y materiales destinados a la enseñanza del E/LE, así como en su decidida apuesta por la investigación y aplicación de las nuevas tecnologías (TIC) — de las que hablaremos más adelante- - Toda su filosofía está recogida en La enseñanza del español como lengua extranjera. Plan Curricular 
del Instituto Cervantes. Este enfoque pedagógico constituyó un primer paso en la tarea de dotar de homogeneidad a las enseñanzas de los distintos centros, "desde un enfoque abierto que permite adecuar las orientaciones generales del plan a las circunstancias particulares de cada uno de los diferentes entornos socioculturales en los que el Instituto desarrolla su actividad", según palabras de Álvaro García de Santa-Cecilia, director académico de aquella institución, en (1995: 80). El Instituto Cervantes emite periódicamente informes sobre El español en el mundo, en donde podemos encontrar una amplia documentación sobre la situación del español - su imparable difusión en todos los continentes - y su enseñanza.

\section{La Situación curricular de la aSignatura de E/LE}

Las Facultades de Letras han estado dedicadas, preferentemente, a los estudios filológicos, históricos y descriptivos, de las diferentes materias lingüísticas y literarias que constituyen el currículo de la Licenciatura en Hispánicas. Las disciplinas lingüísticas se enfocan tradicionalmente desde una perspectiva formal, orientada a la descripción de los sistemas lingüísticos, de sus elementos constituyentes y de las normas que regulan su funcionamiento y utilización. Pero esta concepción, esencialmente estructuralista, se olvida de una de las principales funciones de las lenguas que es la servir de medio de comunicación para los miembros de una comunidad. La sociedad actual demanda una formación y una especialización que no están en condiciones de ofrecer quienes terminan una licenciatura en nuestras Facultades, por lo que no están capacitados para desarrollar su tarea profesional.

La consecuencia práctica de esta orientación generalista y tradicional de nuestras Facultades es que del cómputo total de créditos necesarios para obtener la licenciatur $-300-$, apenas un $10 \%$, o sea 30, están dedicados a materias que se ocupen del análisis del uso de la Lengua, tanto en su modalidad oral como escrita - me refiero ahora a la Universidad de Granada. De estos treinta, solamente 6 créditos se destinan a la asignatura "El español como Lengua Extranjera. Aspectos teóricos y prácticos", que se imparte durante el primer cuatrimestre del curso. Como podemos ver, la situación es totalmente inaceptable y esperamos que este panorama cambie radicalmente en la próxima adaptación de los programas al EEES y al Marco Común de Referencia Europeo - por lo que respecta al E/LE - , lo que supondrá un nuevo modelo de enseñanza superior, que pondrá en marcha metodologías que contemplen sesiones académicas teóricas, teórico-prácticas, prácticas, seminarios, trabajos dirigidos, tutorías especializadas, actividades no presenciales, debates, foros, exposiciones, mesas redondas, comentarios de textos orales, controles de lecturas, sistemas de evaluación específicos, horas de dedicación a la asignatura por parte del alumno, etc.

Para solucionar estas carencias, serán necesarios nuevos esfuerzos para reciclar y actualizar a nuestros licenciados, para que puedan cumplir adecuadamente las nuevas tareas que se les demandan, tanto en la docencia, como en otros puestos profesionales. Los Departamentos organizan Cursos de Doctorado, Jornadas sobre Enseñanzas de Lenguas y Másters para la formación de profesores de Español como Lengua Extranjera, lo que supone una ampliación del tiempo dedicado a la formación de los futuros profesores. 


\section{La FORMACión DEL dOCENTE de E/LE}

La formación de los profesores de E/LE, dependerá, por tanto, de la revisión de los planes de estudio de las universidades, con la incorporación de materias específicas, destinadas a la capacitación de los licenciados para resolver los nuevos retos que esta profesión, en constante auge, está demandando. También dependerá del interés que los alumnos pongan en el perfeccionamiento de su nivel de formación, con la participación en los cursos de metodología y de aplicaciones prácticas que continuamente se programan, tanto por las universidades como por distintos centros públicos y privados. De todas formas, para todo profesor que se inicia en esta interesante tarea, podría constituir un importante punto de referencia, para informarse de lo que ha acontecido en las dos últimas décadas y comprender las motivaciones de las aportaciones más actuales, la consulta de dos documentos clave en toda la revolución de la enseñanza de las lenguas: Un nivel umbral (1979), versión española del Threshold Level de J.Van Ek, elaborada por P.J. Slagter, y el Plan Curricular del Instituto Cervantes (1994), al que ya he hecho referencia con anterioridad, que sigue las recomendaciones de los documentos elaborados por el Consejo de Europa desde finales de los 70, y que han supuesto un avance fundamental en el esfuerzo por definir los principios del enfoque comunicativo y su aplicación a la enseñanza de las lenguas extranjeras.

En relación con el primer documento, Un nivel umbral, el análisis nocio-funcional que lo fundamenta ha sido, en las dos últimas décadas, una de las bases principales del enfoque de los programas de enseñanza de español como lengua extranjera y de los manuales utilizados. Las experiencias obtenidas con su aplicación a las diferentes lenguas, así como la incorporación de nuevas teorías en relación con la adquisición y el aprendizaje de las lenguas, ha hecho que el documento inicial haya ido incluyendo otros aspectos como las estrategias discursivas, el componente sociocultural e intercultural y un componente específico sobre cómo "aprender a aprender", que ha dado lugar a las nuevas aportaciones sobre la "autonomía del aprendizaje".

El PCIC recoge un conjunto de orientaciones e informaciones que sirven de guía para la actividad de los centros del IC en relación con la enseñanza de español, es decir, se incluyen consideraciones sobre la fundamentación teórica del currículo; se especifican los elementos que constituyen los diferentes componentes curriculares: los fines y objetivos, los contenidos, la metodología y la evaluación. En definitiva, y siguiendo sus principios fundacionales, el IC, aplicando un enfoque comunicativo a la enseñanza de la lengua, persigue impulsar una imagen actual de la cultura española y fomentar el intercambio cultural con otros pueblos.

Los contenidos están divididos en cuatro bloques y distribuidos en cuatro niveles; en ellos se incluyen distintas perspectivas de análisis de la lengua y su aprendizaje:

1.- LENGUA Y COMUNICACIÓN: Contenido funcional

2.- LENGUA Y SISTEMA: Contenido gramatical

3.- LENGUA, CULTURA Y SOCIEDAD: Contenido temático

4.- LENGUA Y APRENDIZAJE: Contenido pedagógico.

Estos bloques nos ofrecen inventarios de los distintos elementos necesarios para la enseñanza de la lengua. En el cuarto bloque, Lengua y Aprendizaje, podemos encontrar una lista de estrategias de aprendizaje y comunicación, que facilita la labor del profesor a la hora de programar las actividades de clase, enfocadas al aprendizaje del alumno. 
La metodología nos ofrece orientaciones sobre lo que significa aprender una lengua extranjera, así como consejos prácticos sobre las destrezas lingüísticas y su integración.

En cuanto a la evaluación, establece criterios para cada uno de los niveles del currículo y orientaciones sobre los diversos procedimientos de evaluación.

En resumen, este PCIC está concebido como un plan integrado, donde las decisiones correspondientes a los distintos ámbitos de planificación - objetivos y contenidos, metodología y evaluación-, se relacionan de forma complementaria, por lo que las modificaciones que se introduzcan en cualquier nivel repercutirán en el conjunto.

Sirva este amplio comentario sobre el PCIC - siguiendo la versión de A. García SantaCecilia - para animar, a quienes se inician en la tarea docente, a consultarlo, para aprovechar el inmenso caudal formativo que en él pueden encontrar.

\section{FaCtores importantes a tener EN CUENTA EN LA ENSEÑANZa DEL E/LE}

Tradicionalmente se ha venido prestando atención a un conjunto de factores que mediatizan el papel del profesor y que podríamos considerar como factores externos:

Factores sociales, educativos y culturales:

a) El entorno lingüístico:

- Español como $2^{\mathrm{a}}$ lengua o como lengua extranjera

- Importancia de la lengua en la sociedad-meta

- Actitudes sociales con respecto a la lengua

- El contexto político y social

b) La situación de la enseñanza:

- El punto de partida

- Las características de los alumnos

- La preparación de los profesores

- Los materiales didácticos

- Las características del aula

Pero recientemente se ha despertado un interés considerable por lo que podríamos denominar factores internos, que están relacionados con lo que se viene denominando "el pensamiento o la cognición del profesor" y que, obviamente, tiene importantes repercusiones en el manejo de la clase. Esta corriente se interesa por descubrir los procesos internos de elaboración de pensamiento y de toma de decisiones que el profesor utiliza cuando realiza su trabajo. Por lo tanto, se centra en estudiar hasta qué punto las experiencias previas de aprendizaje del profesor, su conocimiento sobre el tema y sus creencias sobre la enseñanza y el aprendizaje inciden en la práctica de la clase. En consecuencia, estas investigaciones tratan de dar cuenta no sólo de lo que el profesor hace en clase, sino también de por qué hace lo que hace.

D. Freeman - uno de los investigadores más importantes en este campo - señala las consecuencias de este cambio de percepción sobre lo que constituye el proceso de enseñanza: la enseñanza puede incluir dos dimensiones importantes, la cognitiva y la afectiva, que influyen sobre los comportamientos y las acciones que los alumnos y los profesores tienen en clase. 
Freeman plantea también que, si la enseñanza tiene un componente cognitivo, uno se pregunta ¿qué es lo que el profesor sabe?, ¿cómo organiza este conocimiento? o ¿cómo repercute en sus acciones? En consecuencia, la enseñanza ya no consiste sólo en lo que el profesor realiza en clase - perspectiva externa-, sino en saber por qué hace lo que hace - perspectiva interna-.

Veamos algunos aspectos de estos factores internos que atañen al pensamiento del profesor:

\section{1.- La toma de decisiones}

El estudio de los procesos que intervienen en la toma de decisiones de los profesores es uno de los factores fundamentales. El proceso de toma de decisiones se ha dividido en tres fases: la preactiva, la interactiva y la postactiva.

- Las decisiones preactivas se toman antes de que el profesor enseñe, como la planificación de la unidad, la selección de los materiales, etc.

- Las decisiones interactivas se toman cuando el profesor está trabajando con el grupo, como cambiar la actividad programada, reaccionar ante cualquier problema que surja, de disciplina, de comunicación, etc.

- Las decisiones postactivas se toman después de clase, como la selección de nuevas actividades que estén motivadas por las experiencias vividas en las clases anteriores.

No obstante, el estudio de estos procesos no resulta suficiente para conocer el porqué de la actuación de los profesores, ya que no tiene en cuenta su experiencia previa ni las influencias recientes que pueden modificar su conducta. Por eso se han concretado algunos factores que tienen una importancia destacada en la determinación de la manera de enseñar de los profesores:

- La enseñanza que recibió el profesor

- Las creencias del profesor

- Las creencias sobre los alumnos

- El clima afectivo

\section{2.- La enseñanza que se recibe}

Todos nosotros hemos pasado años expuestos a unos determinados modelos de enseñanza, que hemos interiorizado y que, en muchos casos, aun sin quererlo, seguimos empleándolos. Este período de exposición vivido se ha denominado "aprendizaje por observación", que se caracteriza por ser inconsciente; de ahí que los profesores relativamente jóvenes se comporten en clase como les enseñaron a ellos. Según M Kennedy (1991: 16):

El poder del "aprendizaje por observación" y de las imágenes convencionales sobre la enseñanza que derivan de sus experiencias de infancia, hacen que sea muy difícil poder alterar las prácticas docentes y, además, explican, en parte, por qué la enseñanza no ha variado a pesar de décadas de enseñanza reformista.

La investigación sobre el pensamiento del profesor permite que los profesores sean conscientes de sus propias historias y puedan hacer frente a esa tendencia a la imitación del comportamiento de sus profesores. 


\section{3.- Las creencias del profesor}

Es evidente que las creencias que los profesores tienen sobre cómo se aprende una lengua y cómo debería enseñarse, determinan su comportamiento en clase de lengua. Así, si un profesor considera que la gramática y la corrección son objetivos prioritarios de la enseñanza, diseñará programas y tareas para las clases de tipo estructural, donde se prestará una especial importancia al conocimiento del sistema lingüístico. En cambio, si el profesor centra su interés en el empleo de la lengua como instrumento de comunicación, organizará los programas desde un punto de vista funcional o temático y planificará tareas interactivas.

Sucede, sin embargo, con cierta frecuencia, que el profesor es obligado a seguir una determinada metodología, un programa o un libro de texto, con los que no está de acuerdo y cuyo enfoque no encaja en sus creencias de cómo deben aprender las lenguas. Esta situación origina una discrepancia que puede tener graves consecuencias para los alumnos, pues recibirá mensajes confusos que terminarán por desorientarlos.

Conocido, pues, el papel tan importante que ejercen las creencias sobre la labor del profesor, deberíamos preguntarnos ¿de dónde proceden? Parece que intervienen múltiples factores que tienen que ver con las experiencias vividas como alumnos, como profesores y con la formación que han recibido. Por lo tanto, las creencias se originan y se desarrollan en la experiencia y se abstraen de un conjunto de vivencias almacenadas en la memoria a edades muy tempranas. Las experiencias que primero se viven son las que influyen de una manera más decisiva en nuestros juicios finales. En consecuencia, cuanto más pronto incorporemos una creencia a nuestro sistema cultural, más difícil resulta alterarlo. Por ello, las creencias afectan directamente a la percepción y al procesamiento de la información; pero esto no quiere decir que sean inalterables, pues es bien sabido que muchos profesores han cambiado su visión de la enseñanza después de una larga experiencia docente, al comprobar que las cosas pueden ser diferentes de lo que ellos creían y, consecuentemente, han cambiado sus ideas y sus prácticas docentes.

\section{4.- Las creencias sobre los alumnos}

Los profesores no sólo tienen creencias sobre la lengua y su enseñanza, sino también sobre quiénes son sus alumnos. Se ha sugerido que los profesores ven a sus alumnos al menos de siete maneras diferentes y que, además, su forma de considerar a los estudiantes afecta de modo decisivo a sus actividades docentes. R. Meighan y J. Meighan (1990) señalan que los profesores pueden percibir a sus alumnos de siete formas distintas:

- Estudiantes reacios

- Estudiantes receptáculos

- Estudiantes materia prima

- Estudiantes clientes

- Estudiantes compañeros

- Estudiantes exploradores individuales

- Estudiantes democráticos.

Las tres primeras concepciones se corresponden con un tipo de clase muy dominada por el profesor, mientras que las tres últimas reflejarían un punto de vista sobre el aprendizaje más centrado en el alumno. 


\section{5.- El ambiente afectivo}

Este es otro aspecto que ha alcanzado gran importancia en las investigaciones más recientes. Parece demostrado que, para la mayoría de los profesores, crear en clase un ambiente afectivo agradable es un factor prioritario, que puede tener gran trascendencia a la hora de tomar decisiones didáctico-pedagógicas.

Muchos profesores creen que para conseguir ese ambiente afectivo es importante la forma en que se realizan los trabajos, por ello ven el trabajo en parejas o en pequeños grupos como especialmente beneficioso para los estudiantes, puesto que les permite poner en común sus necesidades afectivas y les reporta más beneficios sociales que cualquier otro tipo de trabajo pedagógico.

\section{6.- Materiales para la enseñanza}

Los materiales didácticos son instrumentos fundamentales en el desarrollo del proceso de enseñanza y aprendizaje. Es frecuente que los propios docentes preparen un corpus de materiales, organizados por temas o por niveles, que les permitan preparar las clases con más comodidad y elegir las actividades que resulten más atractivas. Pero, en la mayoría de las ocasiones, se puede contar con libros de texto que, si son bien elegidos - en función del grupo-meta, la metodología adecuada, etc.- - , constituyen una herramienta de trabajo de gran utilidad, en la medida en que proporcionan una solución al problema de la programación del curso, ya que nos ofrecen unidades didácticas desarrolladas, con actividades y ejercicios prácticos, que podemos adaptar a las necesidades, intereses y expectativas de los alumnos de un curso determinado. El manual, por tanto, permite al profesor una cierta seguridad con respecto a la manera de conducir la clase y, al propio tiempo, sirve a los alumnos como material informativo del que pueden disponer en cualquier momento.

Junto a estos materiales, los centros ofrecen también otros servicios complementarios, como bibliotecas, laboratorio de idiomas o centro de recursos informáticos, aparatos magnetofónicos, vídeos, televisión y, en los más actualizados, la utilización de las NTIC (Nuevas Tecnologías de la Información y la Comunicación).

Adjuntamos a continuación algunas direcciones electrónicas que creemos de interés, tanto para profesores como para investigadores en la enseñanza de lenguas:

\section{Centros y grupos de investigación:}

1.Lista de centros universitarios españoles y enlace con sus páginas web: http:// www.rediris.es/recursos/centros/univ.es.html

2.Directorio de Departamentos universitarios españoles de Humanidades: http:// www1.universia.net/catalogaxxi/C10038PPESII1/S10502/INDEX.HTML

3. Listado de centros universitarios hispanoamericanos y enlaces con sus sitios web: http://www1.universia.net/catalogaxxi/C10032PPESII1/S10091/INDEX.HTML

4.Directorios mundiales de universidades: http://www.galilei.com.ar y http:// www1.universia.net/CatalogaXXI/default.asp?IDC=10032\&IDP=ES\&IDI=1

5.Instituto de Filología del C.S.I.C.: http://www.filol.csic.es

6. Seminario de Filología e Informática de la Universidad Autónoma de Barcelona: http:/ /seneca.uab.es/gould/SFI 
7.Grupo de Sintaxis del Español: http://www.sintx.usc.es/EspFrames.html

8.Directorios de hispanistas: http://hispanismo.cervantes.es y http://griso.cti.unav.es/docs/ directorio/principal.html

Foros y listas de distribución:

1.Información y albergue de numerosas listas de distribución profesionales: http:// www.elistas.net/es

2.EcoSEL, lista de distribución sobre el lenguaje y la Sociedad Española de Lingüística: http://www.rediris.es/list/info/ecosel.html

3.InfoLing, lista de distribución de Lingüística Hispánica: http://elies.rediris.es/infoling/ infoling portada.html

4.TecHabla, lista de distribución en castellano sobre tecnologías del habla: http:// www.rediris.es/list/info/techabla.html

5.Hispania, Foro de la Lengua Española: http://www.rediris.es/list/info/hispania.es.html

6.Foro del hispanista del Instituto Cervantes: http://cvc.cervantes.es/foros/default.asp

7.FORMESPA, Foro para profesores de Español como lengua extranjera: http:// formespa.rediris.es/listas.htm

8.ESPAN-L, Foro para profesores de Español: http://www.lsoft.com/scripts/ wl.exe?SL1=ESPAN-L\&H=LISTSERV.TAU.AC.IL

\section{Bibliografías en línea:}

1.Bibliografía de Didáctica del Español Lengua Extranjera: http://cvc.cervantes.es/obref/ bele/busqueda.asp

2.MLA International Bibliography: http://edina.ac.uk/mla [Es preciso estar suscrito]

3.Centro de Información y Documentación del C.S.I.C. (CINDOC): http:// www.cindoc.csic.es [Se necesita estar dado de alta como usuario para acceder al servicio, que conecta con decenas de bases de datos internacionales; LIN-LIT es la base de datos que nos interesa específicamente, disponible en línea y en cederrón (más información a través de sdi@cindoc.csic.es)]

4. Francis, base de datos de estudios sobre Humanidades creada por el CNRS francés: http://www.inist.fr/PRODUITS/francis.php [Se precisa suscripción]

5.Relación de artículos publicados y sumarios de revistas académicas: http:// dialnet.unirioja.es/index.jsp [servicio Dialnet con alertas bibliográficas], http://www.mshreseau.prd.fr/RevuesSom/index.jsp [la Maison des Sciences de l'homme envía sumarios de revistas y permite pedir artículos concretos] y http://sumaris.cbuc.es

6.Compludoc, base de datos de artículos de revistas: http://www.ucm.es/BUCM/complu/ frame.htm

7.Periodical Contents Index Information, base de datos de artículos aparecidos en publicaciones periódicas: http://pci.chadwyck.com [Requiere suscripción]

8.Catálogo de las bibliotecas y archivos del C.S.I.C.: http://www.csic.es/cbic/acceso.htm

9. Bases de datos de tesis doctorales defendidas en España desde 1976: www.csic.es

10. Bases de datos de tesis doctorales y memorias de másters sobre informática y didáctica de las lenguas defendidas en la Universidad de Barcelona: http://www.mcu.es/TESEO 
11. http://www.ub.es/filhis/recopila.html

12. Búsquedas bibliográficas de artículos y monografías en bases de datos: http:// services.inist.fr/public/fre/conslt.htm [Instituto de Información Científica y Tecnológica francés, con una base de datos anterior a 2001]

13. Buscador bibliográfico de Lingüística Hispánica de la Universidad de Barcelona: http://estel.bib.ub.es/filo/index.html

14. Página personal de Joaquim Llisterri: http://liceu.uab.es/ joaquim/home.html [Con excelentes selecciones bibliográficas, sobre todo acerca de Fonética]

Revistas de estudios lingüísticos y sobre didáctica de las lenguas en línea:

1.Enlaces con revistas académicas: http://biblio.colmex.mx/cell/pcentros5.htm, http:// web.prw.net/ vtorres y http://www.letralia.com/itin/revistas/ensayo.htm

2.Proyecto Muse: http://muse.jhu.edu [Agrupa numerosas revistas de Humanidades y Ciencias Sociales, y permite la suscripción electrónica a las mismas]

3.Hispanista. Primera revista electrônica dos hispanistas do Brasil: http:// www.hispanista.com.br

4.Tonos digital. Revista electrónica de Estudios Filológicos: $\underline{\text { http://www.um.es/tonosdigital/ }}$ index.htm

5.Estudios de lingüística del Español: http://elies.rediris.es

6. Cuadernos Cervantes de la Lengua Española: http://www.cuadernoscervantes.com

7. Estudios de Fonética experimental: http://www3.uji.es/ gonzalez/fonetica.pdf

8.Papers de l'Institut Universitari de Lingüística Aplicada: http://www.iula.upf.es/ paperca.htm

9.Glos@s didácticas. Revista electrónica internacional.Didáctica de lenguas y culturas: http://sedll.org/doc-es/publicaciones/glosas

10. Materiales: http://www.sgci.mec.es/usa/materiales/index.shtml

11. Mosaico. Revista para la promoción y apoyo a la enseñanza del español: http:// www.sgci.mecd.es/be/publicaciones/mosaico/mosaico1.htm

12. Núcleo: http://www.revele.com.ve/revistas.php?rev=nucleo\&idm=id0sub3

13. Porta linguarum. Revista Interuniversitaria de Didáctica de las lenguas: http:// www.ugr.es/ portalin/

14. Quaderns digitals: http://www.quadernsdigitals.net/

15. Revista RedEle: http://formespa.rediris.es/revista.htm

16. http://www.octaedro.com/espanoleninternet/webs.html

17. http://www.octaedro.com/espanoleninternet/Bibliografia/BIBLIOGRAF.HTML

18. http://www.campusred.net/escuelanntt/principal.htm

19. http://www.rediris.es/list/info/dilenguas.es.html

20. http://elies.rediris.es/infoling/

21. http://liceu.uab.es/ joaquim/applied linguistics/new technologies/LengEsp Materiales WWW.html

22. http://liceu.uab.es/ joaquim/applied linguistics/new technologies/ELE Internet Bib.html

23. http://www.tierradenadie.de/seminario/index.htm 
Centros virtuales o sitios con recursos

1.Instituto Cervantes y Centro Virtual Cervantes: http://www.cervantes.es y http:// cvc.cervantes.es/.

2.Centro Virtual de Recursos María Moliner: http://cvc.cervantes.es/obref/bele/busqueda.asp

3.Elenza: http://redgeomatica.rediris.es/elenza/ [Página de la Consejería de Educación en Australia y Nueva Zelanda con numerosos materiales y recursos]

4.Base de datos 2000, de Mar Cruz Piñol: http://www.octaedro.com/ele

5.Webs mencionadas por Cruz Piñol [2002] y útiles para el ELE: http://www.octaedro.com/ espanoleninternet/webs.html

6.Unidad en la diversidad, servicio informativo sobre la lengua española: http:// www.unidadenladiversidad.com/

7. Cultura e intercultura en la enseñanza del Español como Lengua Extranjera, de Emma Martinell, Mar Forment y Mar Cruz Piñol: http://www.ub.es/filhis/culturele/index.html

8.Información sobre el Marco de Referencia Europeo y el Portfolio para las lenguas: http://cvc.cervantes.es/obref/marco/, http://www.fle.fr/pro/besancon/forum.html, http:// comunidad-escolar.pntic.mec.es/745/info6.html, http://aplicaciones.mec.es/programas-europeos/ jsp/plantilla.jsp?id=pel d, http://www.eeooiinet.com/portfolio/triptico portfolio.pdf, http:// www.sgci.mec.es/be/media/pdfs/mosaico/Mosaico09.pdf, http://redgeomatica.rediris.es/elenza/ documentos/index.html y http://www.encuentro-practico.com/pdf03/cassany.ppt

9. Bases de datos sintácticos del español actual: http://www.bds.usc.es

10. Página de Joaquim Llisterri con materiales para la enseñanza y el aprendizaje del ELE: http://liceu.uab.es/ joaquim/home.html

11. Página sobre Didáctica del ELE: http://es.geocities.com/didacticadelespanol/

12. Zona ELE: http://www.zonaele.com/

Editoriales especializadas en ELE

1.Publicaciones del CIDE del Ministerio de Educación: http://www.mec.es/cide/ publicaciones/pub cide.htm

2.Anaya: http://www.anaya.es/home.html

3.Difusión: http://www.difusion.com/ele/inicio

4.Edelsa: http://www.edelsa.es/

5.Edinumen: http://www.edinumen.es/

6. Espasa: http://www.integralhost.com/espasa/nuevaweb/productos.asp?id=39\&idcol=172

7. RedEle, colección digital de memorias, tesis e investigaciones sobre didáctica del ELE: http://formespa.rediris.es/biblioteca.htm

8.SGEL: http://www.sgel.es/espanyol/ieindex.htm

\section{REFERENCIAS BIBLIOGRÁFICAS}

Freeman, D. Y Richards, J. (eds.) (1996). Teacher Learning in Language Teaching, New Cork: Cambridge University Press.

García De Santa-Cecilia, A. (1995). El curriculo de español como lengua extranjera. Madrid: Edelsa. 
Instituto Cervantes (1994). La enseñanza del español como lengua extranjera. Plan curricular del Instituto Cervantes. Madrid: Publicaciones del Instituto Cervantes.

Kennedy, M. (1991). An Agenda for Research on Teacher Learning. East Lancing, Mich.: National Center for Research on Teacher Learning.

Meighan R. y Meighan, J. (1990). "Alternative roles for learners with particular reference to learners as democratic explorers in teacher education courses", en The School Field, 1 (1): 61-67.

Melero Abadía, P. (2000). Métodos y enfoques en la enseñanza laprendizaje del español como lengua extranjera. Madrid: Edelsa. (Para una visión general de los trabajos sobre el español se puede consultar el Vademécum para la formación de profesores Obra dirigida por J Sánchez Lobato e I. Santos Gargallo, Madrid, SGEL, 2004. También se pueden consultar las Actas del Congreso de la Lengua Española, Instituto Cervantes, Madrid, 1994).

Sánchez Pérez, A. (1992). Historia de la enseñanza del español como lengua extranjera. Madrid: SGEL.

Slagter, P. (1979). Un Nivel Umbral. Estrasburgo: Consejo de Europa. 